\title{
Parasitic infections of the lung: a guide for the respiratory physician
}

\author{
H Kunst, ${ }_{1}^{1}$ D Mack, ${ }^{2}$ O M Kon, ${ }^{3}$ A K Banerjee, ${ }^{4}$ P Chiodini, ${ }^{2,5}$ A Grant ${ }^{5}$
}

'Department of Respiratory Medicine, Birmingham Heartlands Hospital, Birmingham, UK

${ }^{2}$ Department of Clinical Parasitology, Hospital for Tropical Diseases, London, UK ${ }^{3}$ Department of Respiratory Medicine, St Mary's Hospital, Imperial College Healthcare NHS Trust, London, UK

${ }^{4}$ Department of Radiology, Birmingham Heartlands Hospital, Birmingham, UK ${ }^{5}$ Department of Clinical Research, London School of Hygiene and Tropical Medicine and Hospital for Tropical Diseases, London, UK

\section{Correspondence to} Heinke Kunst, Department of Respiratory Medicine, Birmingham Heartlands Hospital, Bordesley Green East, Birmingham B9 5SS, UK; hkunst@doctors.org.uk

Received 29 November 2009 Accepted 28 May 2010 Published Online First 29 September 2010

\section{ABSTRACT}

Parasitic infections of the lung occur worldwide among both immunocompetent and immunocompromised patients and may affect the respiratory system in a variety of ways. This review provides an update on the presenting symptoms, signs, investigation and management of diseases affecting the lung caused by protozoa, nematodes and trematodes. The clinical presentations and radiographic findings of several of these diseases may mimic tuberculosis and malignancy. It is important to consider parasitic infections in the differential diagnosis of such lung diseases. If identified early, most parasitic diseases that affect the lung are curable with medical or surgical treatments.

\section{INTRODUCTION}

With increasing travel and migration, rates of parasitic lung and pleural diseases are increasing in the immunocompetent population in developed countries as well as among immunocompromised patients. Respiratory physicians should consider parasitic diseases in the differential diagnosis of lung conditions such as tuberculosis and malignancy, with which parasitic lung diseases may be confused.

This review describes the presentation, investigation and management of common parasitic infections affecting the lung caused by protozoa, nematodes and trematodes. The diseases have been grouped according to their manner of presentation: (1) those presenting with focal lesions and (2) those which characteristically present with diffuse lung disease. Focal lung lesions have been divided into cystic lung lesions, coin lesions and consolidation/ pleural effusion. Diffuse lung disease has been divided into transient pulmonary infiltrates and alveolar/interstitial lung changes. Diseases that may present in a variety of ways are fully described only the first time they are mentioned.

\section{CONDITIONS PRESENTING WITH FOCAL LUNG LESIONS \\ Cystic lung lesions \\ Hydatidosis \\ Distribution and life cycle}

Hydatid disease is caused by larvae of Echinococcus tapeworm species, the definite hosts of which are members of the Canidae family (dogs and foxes). Most cases are caused by Echinococcus granulosus which has a worldwide distribution including South America, countries surrounding the Mediterranean, the Middle East, some sub-Saharan African countries, Russia and China. Although most cysts form in the liver, $20-30 \%$ form in the lung.
Dogs are the definitive host for E granulosus and harbour the adult worms in their gut. The eggs shed in dog faeces remain viable for many weeks and are able to contaminate food sources of intermediate hosts such as sheep, cattle and horses. When humans become accidental intermediate hosts after eating food contaminated with eggs, the ingested eggs hatch, releasing larvae which migrate from the gastrointestinal tract to the circulation. The eggs travel to the liver or lungs and slowly develop into hydatid cysts over a period of several months or years. Occasionally, lung cysts form after transdiaphragmatic spread of parasites following the rupture of liver cysts.

\section{Presentation}

Primary infection is asymptomatic and patients may remain asymptomatic for years, during which time lung lesions may be discovered incidentally on a chest $\mathrm{x}$-ray. In Europe the average patient is in their 30 s at diagnosis. ${ }^{1}$ Cysts may cause symptoms by compression of adjacent structures, and lung cysts may present with chest pain, cough, haemoptysis or pneumothorax. Symptoms may also occur if antigenic material is released from the cyst, causing a hypersensitivity reaction with fever, wheeze and urticaria and, rarely, anaphylaxis. Cysts may become secondarily infected causing empyema or lung abscess formation.

\section{Imaging features}

Cysts can be seen as single or multiple well-defined homogenous lesions surrounded by otherwise normal lung parenchyma on a plain chest x-ray. The lower lobes, posterior lung segments and the right lung are affected most frequently. ${ }^{2}$ The cyst wall often calcifies over time. A CT scan of the chest may reveal further diagnostic features including collapse of the laminated membrane from the surrounding host tissue, the presence of daughter cysts and the presence of cyst rupture. ${ }^{3}$ Air between the host tissue and laminated membrane of ruptured cysts may produce crescentshaped lucencies. A pleural effusion or a hydropneumothorax may develop following the rupture of a pulmonary cyst into the pleural space. Collapsed laminated membrane may float in the cyst cavity, producing the 'water lily' sign (figures 1 and 2). ${ }^{34}$

\section{Laboratory diagnosis}

Peripheral blood eosinophilia may be found in no more than $50 \%$ of cases. A substantial rise in eosinophil count is often associated with a leakage of antigenic material from the cyst. Serological tests to support the initial diagnosis are available at reference laboratories but are less sensitive for the diagnosis of lung disease than for hepatic disease. ${ }^{1}$ 


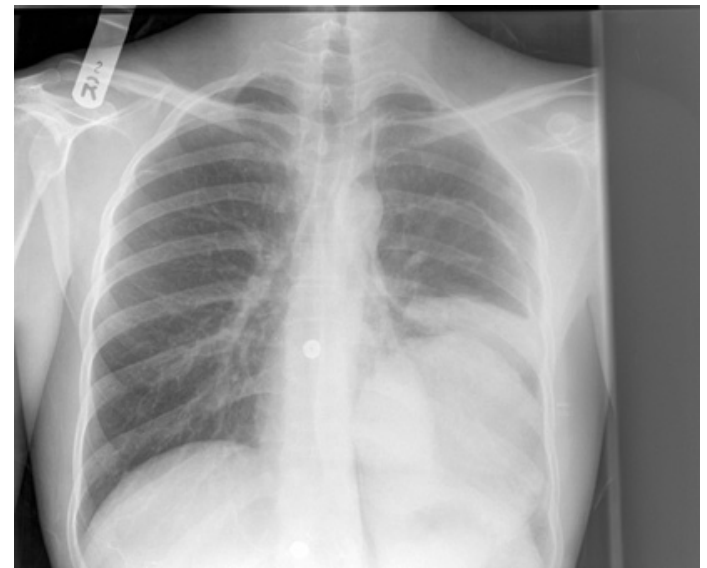

Figure 1 Chest $x$-ray showing a hydatid cyst in the left lower lobe with a collapsed hydatid cyst exhibiting the 'lily pad' sign lying above it.

Postoperatively, serological tests may be used to monitor the immunological response to treatment. ${ }^{5}$

While percutaneous aspiration of liver cysts under ultrasound guidance can support the diagnosis by demonstrating the presence of protoscolices or hooklets, this is not generally recommended because of the risk of leakage resulting in an anaphylactic reaction.

\section{Management}

In a small number of cases hydatid cysts resolve spontaneously. ${ }^{6}$ In the majority of cases the treatment of choice is surgical excision of cysts with a view to preserve as much lung parenchyma as possible. ${ }^{7}$ Albendazole should be avoided preoperatively as it may soften the cyst wall and increase the chance of rupture. It may be given as adjunctive therapy once the cyst has been removed. ${ }^{8}$ When surgery is contraindicated or not feasible in patients with multiorgan involvement, medical treatment with albendazole with or without praziquantel is recommended. ${ }^{9}$ Expert advice should be sought regarding chemotherapeutic regimens before and after surgery and for cases where surgery is not to be carried out. While aspiration followed by injection of a scolicidal agent and re-aspiration (PAIR) has been used as a therapeutic tool when surgery is contraindicated, ${ }^{10}$ the World Health Organization states that PAIR is contraindicated for lung cysts ${ }^{11}$ and the authors endorse that view.

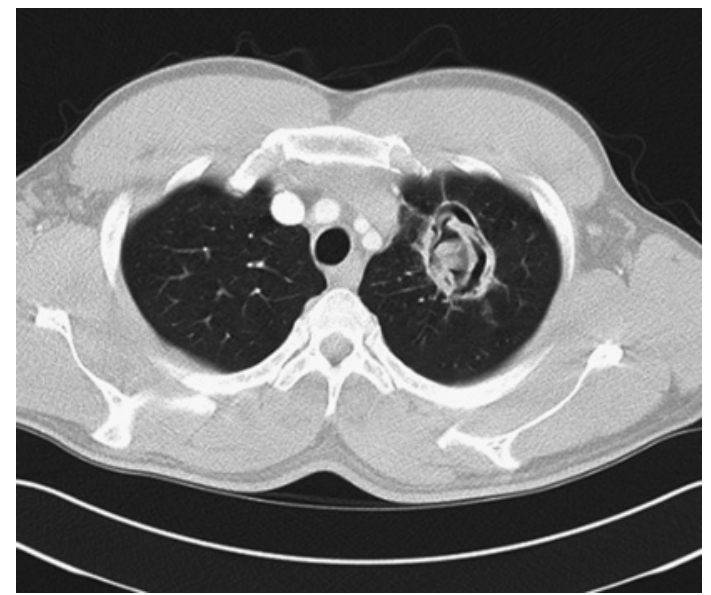

Figure 2 CT scan of the thorax showing a ruptured hydatid cyst in the left upper lobe of the lung.

\section{Coin lesions \\ Dirofilariasis \\ Distribution and life cycle}

Pulmonary dirofilariasis is caused by the dog heartworm Dirofilaria immitis and has been reported from Japan, Australia, the USA and Italy ( $D$ repens). Adult worms live in the right ventricle of the definitive canine hosts and produce circulating microfilaria which can be transmitted by a variety of mosquito species to humans. In humans the worms pass through the right ventricle but fail to mature and are swept away to peripheral pulmonary arteries.

\section{Presentation}

Most patients are asymptomatic, although symptoms including chest pain, cough, haemoptysis, wheezing, fever, chills and malaise have been reported. ${ }^{12}$

\section{Imaging features}

The most common presentation is with a coin lesion on chest radiography. These are usually $1-3 \mathrm{~cm}$ in diameter and sharply defined. In established lesions a central necrotic area is surrounded by a granulomatous reaction and fibrous wall. ${ }^{13}$ Dead worms may calcify. Positron emission tomographic scanning may show an increased uptake of fluorodeoxyglucose around necrotic nodules, a feature also seen in other infectious lung nodules. ${ }^{14}$

\section{Laboratory diagnosis}

Eosinophilia may be present in a small number of patients. There are no reliable serological tests available commercially. Most cases are diagnosed by identifying the worm in biopsy specimens after thoracatomy for a pulmonary nodule thought to be a malignancy. ${ }^{15}$

\section{Management}

Chemotherapy is ineffective. The only treatment is surgical excision if indicated.

\section{Consolidation/pleural effusion \\ Paragonimiasis \\ Distribution and life cycle}

Pulmonary paragonimiasis is most frequently seen in south east Asia but also occurs in central Africa and South America. It is caused by lung flukes of the genus Paragonimus. Paragonimus westermani is responsible for most cases. The adult worms are found in pulmonary cysts, usually in pairs. Adult worms produce eggs which are secreted in sputum or faeces. Humans are usually infected by eating the second intermediate host, fresh water crab or crayfish, if raw or lightly cooked. After ingestion the larvae penetrate the intestinal wall and migrate to the lung parenchyma. They mature in a fibrous host-derived capsule, usually in the upper zones of the lung.

\section{Presentation}

Presenting symptoms include pleuritic chest pain, haemoptysis, chronic cough and fever. If pulmonary cysts erode into adjacent bronchi, patients may suffer life-threatening haemoptysis. ${ }^{16}$

\section{Imaging features}

Radiographic features of paragonimiasis include patchy air space consolidation, cystic changes and ring shadows. Pleural effusions and pneumothoraces may occur. On the CT scan round lowattenuation cystic lesions filled with fluid or gas may be seen within an area of consolidation. Peripheral linear opacities seen on chest x-rays are suggestive of worm migration tracks on corresponding CT scans. Intracystic worms may be detected on a CT scan of the thorax. ${ }^{17} 18$ Patients with pulmonary 
paragonimiasis who present with parenchymal lesions on a CT scan of the thorax may have bronchial stenosis on bronchoscopy. ${ }^{19}$ Paragonimiasis is often misdiagnosed as tuberculosis or malignant disease and should be considered in the differential diagnosis of these conditions in those who have lived in endemic countries. $^{20-22}$

\section{Laboratory diagnosis}

Peripheral blood eosinophilia is mainly seen during larval migration through the lung and total serum IgE may be raised. The eosinophil counts are usually elevated in pleural and bronchoalveolar lavage fluids. The identification of the characteristic operculated eggs in faeces, sputum or bronchoalveolar lavage fluid confirms the diagnosis. Specific serological testing is not performed in the UK but samples may be referred to overseas laboratories for specific IgG and IgM antibody testing when paragonimiasis is suspected. ${ }^{20} 23$

\section{Management}

Medical treatment is usually with praziquantel. Some patients may require surgery for persistent pneumothorax, pleural effusion or empyema. ${ }^{24}$

\section{Amoebiasis \\ Distribution and life cycle}

Amoebiasis is caused by Entamoeba histolytica, a protozoan found worldwide. E histolytica is the causative agent of invasive amoebiasis but is morphologically indistinguishable from a related but non-pathogenic species, E dispar. E histolytica is endemic in regions with poor sanitation and poor socioeconomic conditions. The motile trophozoite forms of the parasite live in the lumen of the large intestine where they multiply and differentiate into the cyst forms. Cysts are passed in the faeces and are highly resistant to environmental conditions, facilitating faecal-oral transmission. Ingested cysts are transformed into trophozoites.

\section{Presentation}

In most infections the organism does not invade the gut mucosa. In a subset of cases the E histolytica trophozoites bind to intestinal epithelial cells via a lectin expressed on the parasite surface. This leads to lysis of the epithelial cells and invasive amoebiasis resulting in colitis. Blood-borne spread of the parasite may lead to the formation of an amoebic liver abscess which may present several months after the initial infection when diarrhoea may be absent.

Amoebic liver abscess may cause irritation of the diaphragm and the patient may present with cough. A serous pleural effusion may occur in conjunction with an amoebic liver abscess and does not indicate extension of the disease. ${ }^{25}$ Amoebic liver abscess may also present with chest pain due to hepatic enlargement and inflammation. Elevation of the hemidiaphragm can cause segmental atelectasis of the lung and lead to secondary bacterial pneumonia. ${ }^{26}$

Amoebic pleuropulmonary disease is the most common complication of amoebic liver abscess, occurring in $15 \%$ of patients with amoebic liver disease and in $1 \%$ of patients with amoebic dysentery. ${ }^{27}$ It most commonly occurs by direct extension from a superior right lobe hepatic abscess through the diaphragm into the right lower lobe of the lung, presenting with cough, pleuritic pain and dyspnoea. ${ }^{25}$ Pleuropulmonary amoebiasis may also occur following haematogenous spread of organisms to the lungs or lymphatic spread from the liver to the diaphragm. Intrathoracic complications include rupture of an amoebic liver abscess into the pleural cavity with empyema formation which carries a mortality of $15-35 \% .^{25}$ Infiltration of the lung parenchyma may result in pneumonia or lung abscess formation. Furthermore, a hepatobronchial fistula leading to expectoration of 'anchovy sauce-like' purulent sputum can develop. Rarely, a bronchobiliary fistula may occur causing bile expectoration

\section{Radiographic features}

Common radiological features include right pleural effusion and basal lung disease. Right lower lobe consolidation may develop, which may progress to abscess formation (figure 3).

\section{Laboratory investigations}

Eosinophilia is not a feature of amoebic infections. If symptoms of dysentery are present, freshly passed stools should be examined for amoebic trophozoites. Trophozoites are seldom found in sputum, pleural fluid or abscess aspirates. Antibodies directed against the parasite may be detected by a variety of methods. Antibody tests start to become positive after approximately 1 week and have a sensitivity of $95 \%$ for the diagnosis of amoebic liver abscess but are only positive in approximately $75 \%$ of cases of intestinal infection. The E histolytica adhesin may be detected in faecal specimens by ELISA. This test is also helpful in differentiating between $E$ histolytica and $E$ dispar in asymptomatic patients who pass amoebic cysts.

\section{Management}

Medical treatment consists of a course of tinidazole or metronidazole to kill trophozoites in tissue, followed by diloxanide furoate or paromomycin to eliminate cysts from the intestinal lumen. ${ }^{28} 29$ Surgical treatment of pulmonary amoebiasis may be required when there is direct pulmonary involvement or spread to pleura.

\section{CONDITIONS PRESENTING WITH DIFFUSE LUNG DISEASE Transient pulmonary infiltrates \\ Ascariasis \\ Distribution and life cycle}

Ascariasis is caused by Ascaris lumbricoides, the round worm. It is common worldwide in areas with poor sanitation where there is faecal contamination of soil or food. Transmission of the disease is faecal-oral. After eggs are ingested they hatch and larvae migrate via the portal circulation to the liver then via the heart to reach the lungs 2 weeks after ingestion. Larvae then ascend to the trachea, are swallowed and eventually develop into adults in the small intestine, producing eggs 10-12 weeks after ingestion.

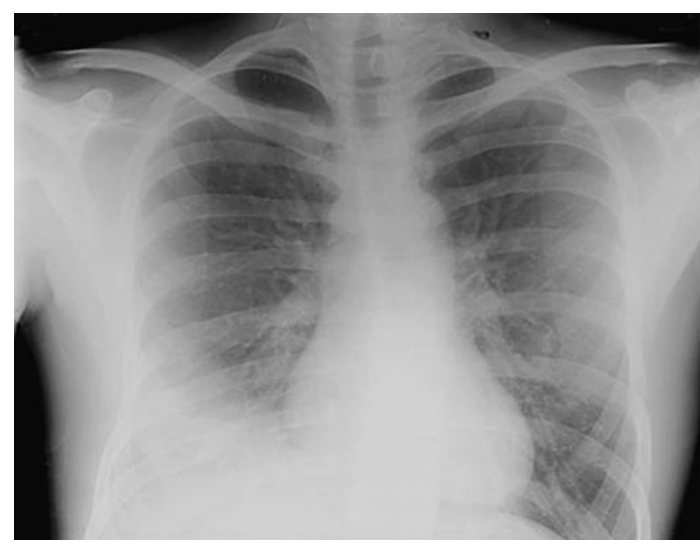

Figure 3 Chest $x$-ray showing right basal consolidation and pleural effusion secondary to amoebic liver abscess. 


\section{Presentation}

During the second week of infection, as the larvae invade the lung tissue, a small proportion of patients become symptomatic. Loeffler's syndrome is caused by larval migration into the alveoli which triggers an allergic response leading to respiratory symptoms including cough, wheeze, dyspnoea, chest pain, fever and haemoptysis. The illness usually resolves spontaneously after several weeks.

\section{Imaging features}

During pulmonary migration, transient nodular or diffuse pulmonary infiltrates on the chest x-ray support the diagnosis of Loeffler's syndrome. Chronic eosinophilic pneumonia has been associated with ascariasis of the lung. ${ }^{30}$ Spontaneous pneumothoraces have been described infrequently. ${ }^{31}$

\section{Laboratory diagnosis}

During larval migration a leucocytosis and peripheral blood eosinophilia are often present and total serum IgE may be elevated. Eosinophils and larvae may be found in sputum. The diagnosis is confirmed by finding characteristic Ascaris eggs in the stool on microscopy, although these may not be passed until after resolution of the pulmonary symptoms. Serological tests are not routinely available.

\section{Management}

A single dose of albendazole is the treatment of choice. Mebendazole, piperazine and pyrantel pamoate are alternatives. ${ }^{28}$

\section{Hookworm infections Distribution and life cycle}

Ancylostomiasis is common in tropical and subtropical countries, caused by two species of hookworm (Ancylostoma duodenale and Necator americanus). A duodenale is also found in the Middle East and southern Europe; $N$ americanus is found sporadically in the southeastern USA. Hookworm eggs are passed in faeces and develop in soil into filariform larvae able to penetrate the skin of the human host. Poor sanitation and the absence of footwear favour transmission. The larvae penetrate blood vessels and undergo heart-lung migration before breaking out into the alveoli and ascending to the pharynx from where they are swallowed. The hookworms subsequently mature into adults in the small intestine. Eggs first appear in the stool 5 weeks after skin invasion.

\section{Presentation}

Pulmonary manifestations are usually mild. During larval migration in the lung the hookworm may cause symptoms and signs consistent with Loeffler's syndrome including dry cough, wheeze, dyspnoea and fever.

\section{Imaging features}

The radiographic changes are consistent with Loeffler's syndrome and an eosinophilic pneumonia with transient non-segmental areas of consolidation. ${ }^{13}$

\section{Laboratory diagnosis}

Peripheral blood eosinophilia is found during larval migration and commonly persists during gastrointestinal infection. Although their appearance may be delayed, finding eggs in the stool supports the diagnosis. Serological tests are not useful in view of many cross-reactions with other helminthic infections. ${ }^{27}$

\section{Management}

Recommended treatment consists of a single dose of albendazole. Mebendazole and pyrantel pamoate are alternatives. ${ }^{28}$

\section{Toxocariasis}

\section{Distribution and life cycle}

Human toxocariasis is usually caused by the dog round worm Toxocara canis which commonly infects juvenile dogs and has a worldwide distribution. Humans, who are incidental hosts, are usually infected by the ingestion of eggs. The disease primarily affects children, who acquire the infection through contact with soil contaminated with embryonated eggs. Fresh animal faeces are not infectious as the eggs require $2-3$ weeks to embryonate in the soil. Ingested eggs hatch in the stomach. Larvae penetrate the mucosa of the gut and enter the mesenteric vessels from where they migrate to the lungs and other organs.

\section{Presentation}

Most infections occur in children and most are asymptomatic. Patients may present with immunologically-mediated symptoms including cough, dyspnoea and wheeze which may present as asthma or bronchitis. Presenting signs may include hepatomegaly, splenomegaly and ocular lesions.

\section{Radiographic features}

Radiological features may include transient localised changes or widespread patchy areas of consolidation. ${ }^{13}$

\section{Laboratory diagnosis}

Toxocara larvae do not develop into adults in the human host, so eggs are not passed in human faeces. Visceral larva migrans is characterised by persistent peripheral eosinophilia and leucocytosis. A high degree of eosinophilia in the differential cell count of bronchoalveolar lavage fluid may support the diagnosis. ${ }^{32} 33$ Serological tests are available at reference laboratories. Although positive Toxocara serology supports a diagnosis of visceral larva migrans, titres may remain elevated for years and other causes of illness should be considered. ${ }^{34}$ Children suspected of ingesting infective Toxocara eggs may be investigated with full blood count and Toxocara serology tests at baseline and at 3 months. In practice this is seldom required as, in many cases where dog faeces have been ingested, it is possible to establish that the sample is relatively fresh and thus not likely to contain embryonated eggs. Tissue biopsy is not required for the diagnosis of visceral larva migrans.

\section{Management}

Ocular disease is treated under the supervision of an ophthalmologist with local or systemic steroids. Anthelminthics are not usually given. Visceral larva migrans is treated with albendazole. Corticosteroids may be required in more severe cases. ${ }^{28}$

\section{Alveolar/interstitial changes \\ Schistosomiasis \\ Distribution and life cycle}

Schistosomiasis is endemic throughout the tropics and it is estimated that more than 200 million people worldwide are infected. ${ }^{35}$ There are three main species of schistosomes: Schistosoma mansoni, Schistosoma haematobium and Schistosoma japonicum. The adult trematode worms are found in the vesical plexus (S haematobium) or in the mesenteric veins (S mansoni and $S$ japonicum). Their eggs are excreted in urine or faeces respectively. Humans are infected by cercariae during contact with fresh water. The organisms enter the circulation and pass through the heart, lungs and then the liver to reach the target venous plexus. There the worms mature and mate, releasing eggs 4-6 weeks after skin penetration. The initial release of eggs may give rise to acute schistosomiasis which occurs predominantly in non-immune hosts. Eggs that are not passed into the 
bladder or intestinal lumen are the main cause of chronic disease, causing granulomatous reactions and fibrosis. In severe longstanding $S$ mansoni and $S$ japonicum infections, the development of hepatosplenomegaly and portal hypertension may lead to diversion of eggs to the lung vasculature. This results in obliterative arteritis which may cause pulmonary hypertension.

\section{Presentation}

In non-endemic countries, returned non-immune travellers may present with acute schistosomiasis while migrants from endemic countries may present with chronic disease. Acute schistosomiasis (Katayama fever) may present with fever, cough, dyspnoea, rash and arthralgias. ${ }^{36}$ The precise pathogenesis of Katayama fever is unknown, but it is thought to be due to an immune complex phenomenon initiated by eggs laid by maturing schistosomes. Typically, symptoms occur 4-6 weeks after infection. In acute schistosomiasis, serological tests are often negative and eggs are not detectable, requiring the diagnosis to be made on clinical and epidemiological grounds. In heavy infections, the migratory phase through the lungs may produce a pneumonitis resembling that produced during hookworm migration. A Loeffler-like syndrome may occur during the treatment of heavy infections. Acute symptoms are usually self-limiting. In the chronic stage the commonest symptoms are dyspnoea, reduced exercise tolerance and chest pain. Clinical signs of severe hypoxaemia such as digital clubbing may be seen. Since schistosomiasis is an important cause of pulmonary hypertension, it should always be considered as a differential diagnosis in patients from endemic countries. ${ }^{37}$ The chronic form of schistosomiasis may also cause pulmonary arteriovenous fistulas. ${ }^{38}$

\section{Radiological features}

Nodular ill-defined lesions or reticulonodular changes may be seen on the chest x-ray in acute pulmonary schistosomiasis (figure 4). ${ }^{39}$ Although radiological findings are usually transitory in acute infection, chest $\mathrm{x}$-rays may show features resembling granulomatous disease or tuberculosis. ${ }^{40} 41$ In chronic lung disease, interstitial or granulomatous changes are caused by the widespread deposition of eggs in the pulmonary vasculature. A CT scan of the thorax may show nodular changes and ground glass opacification (figure 5 ). ${ }^{42}$

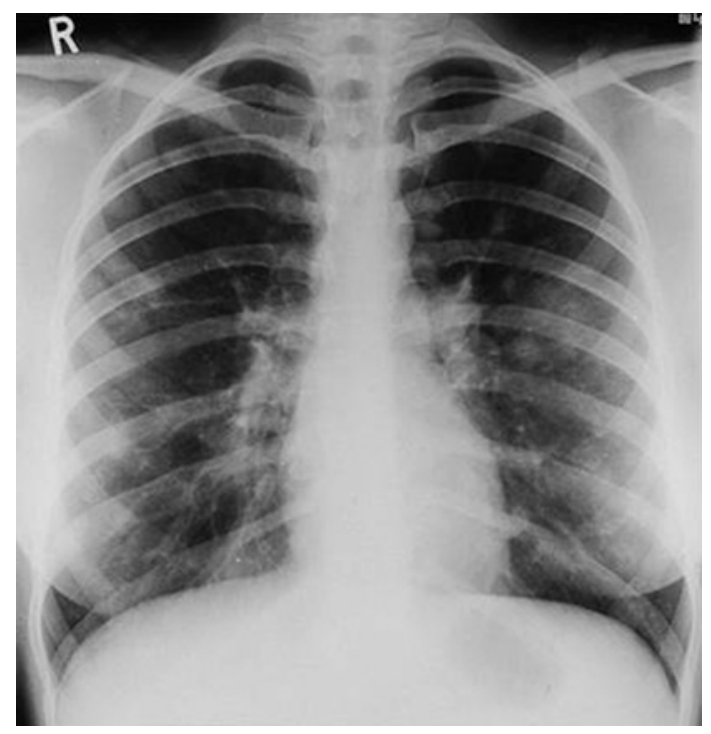

Figure 4 Chest x-ray showing widespread pulmonary nodules in pulmonary schistosomiasis. Reproduced with permission from Waldman et al. ${ }^{42}$

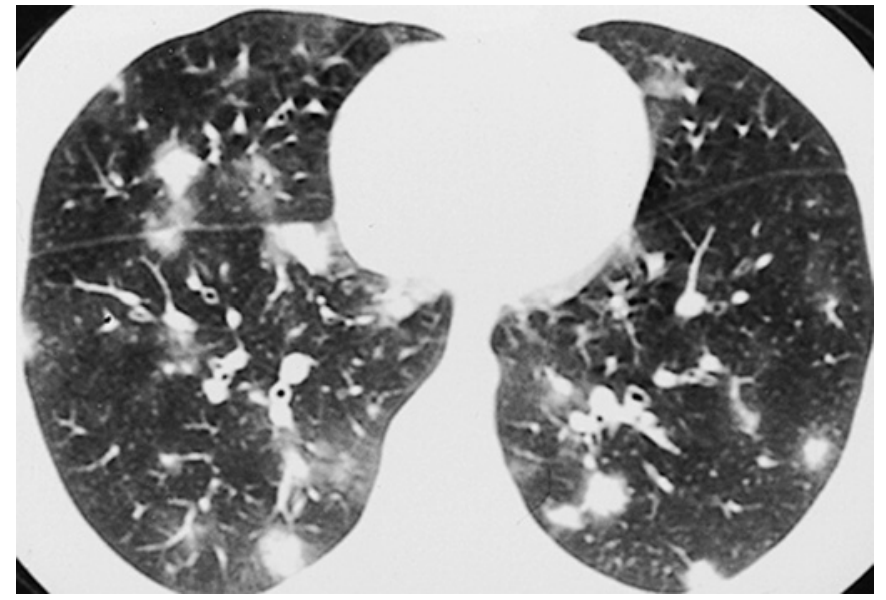

Figure 5 CT scan of the thorax showing the halo sign in pulmonary schistosomiasis.

\section{Laboratory investigation}

Peripheral blood eosinophilia is present in more than $65 \%$ of patients. ${ }^{43}$ Eggs of the Schistosoma species may be found in faeces, urine and sputum or bronchoalveolar lavage 6 weeks after infection. Eggs of $S$ haematobium are most likely to be found in late morning urine samples. ${ }^{44}$ Terminal urine specimens maximise sensitivity. ${ }^{45}$ IgG antibodies to Schistosoma egg antigen are detectable 6-12 weeks following infection and can be measured by ELISA. Such assays have sensitivities of $>90 \%$ for the diagnosis of schistosomiasis. ${ }^{46}$ These tests may remain positive for several years and are not helpful for monitoring response to treatment. Serological testing in asymptomatic travellers should be delayed for a period of 3 months following exposure to fresh water to allow time for seroconversion. Schistosomal serology may be negative, especially in Katayama fever, but if positive it provides helpful diagnostic information. ${ }^{43} 47$

\section{Management}

The treatment of choice for schistosomiasis is praziquantel. ${ }^{28} 48$ In acute schistosomiasis (Katayama fever) praziquantel is administered under steroid cover. A second course of praziquantel may need to be given 3-6 months later to eradicate any schistosomes that may have survived the first course of treatment. ${ }^{47}$ Treatment is less beneficial in advanced chronic disease.

\section{Strongyloidiasis}

\section{Distribution and life cycle}

Strongyloidiasis is caused by the nematode Strongyloides stercoralis and is prevalent in tropical and subtropical countries. Filariform larvae penetrate the skin, enter blood vessels and undergo heart and lung migration. They migrate into alveoli and subsequently ascend to the trachea. Larvae are swallowed and develop in the small intestine into adult worms which produce eggs. These are deposited in the intestinal mucosa where they hatch into rhabditiform larvae which are excreted in faeces. Occasionally, rhabditiform larvae mature to the filariform stage while still in the bowel lumen. These filariform larvae may penetrate the bowel wall or the perianal skin to initiate a new cycle (autoinfection). Thus, in the absence of treatment, an individual may harbour Strongyloides for several decades.

\section{Presentation}

Clinically the infection is often asymptomatic, but mild symptoms may occur in the initial stages of lung migration. In the immunocompromised patient (including those on steroid 


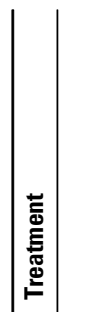

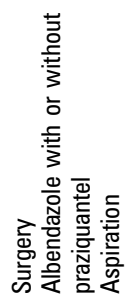
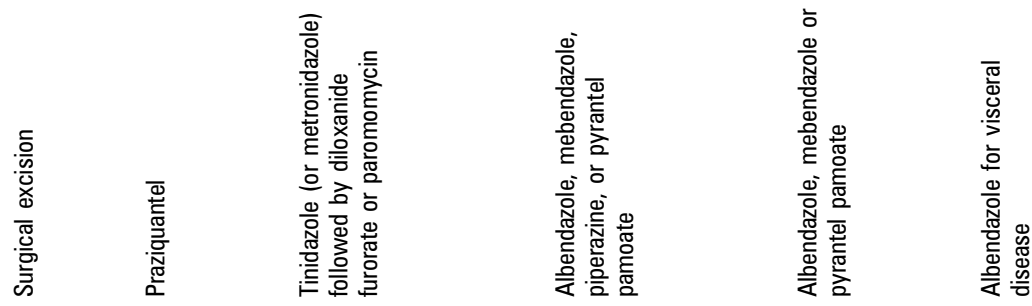

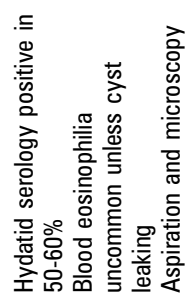

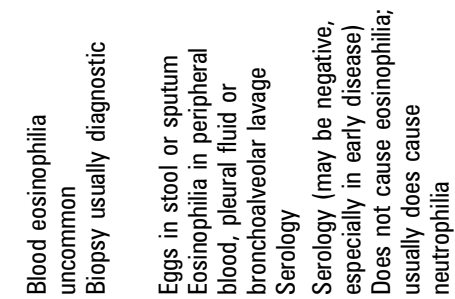

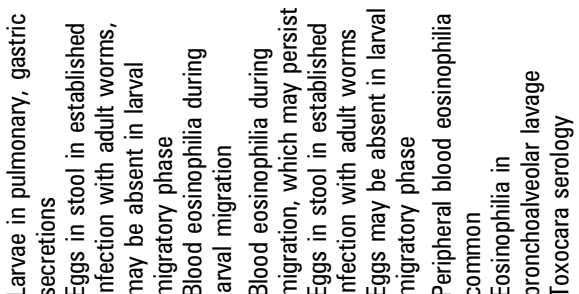
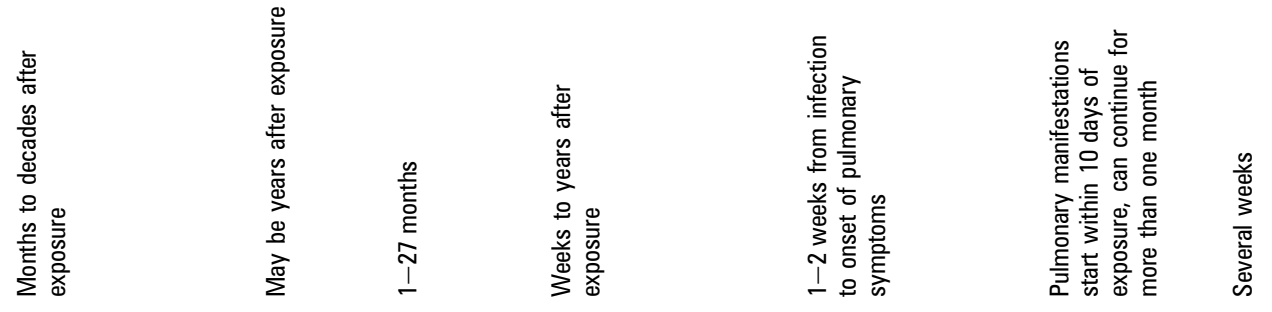

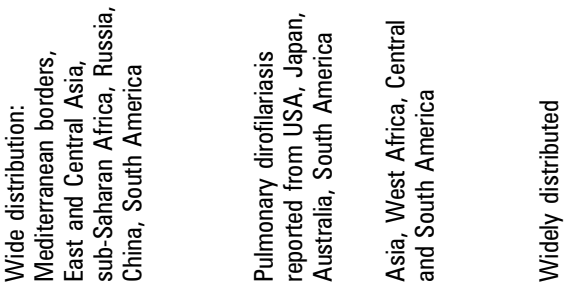

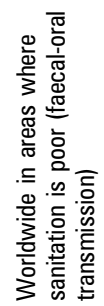

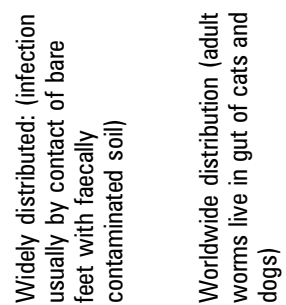

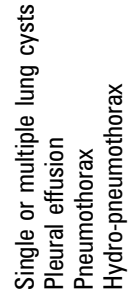

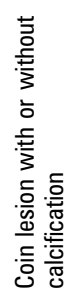
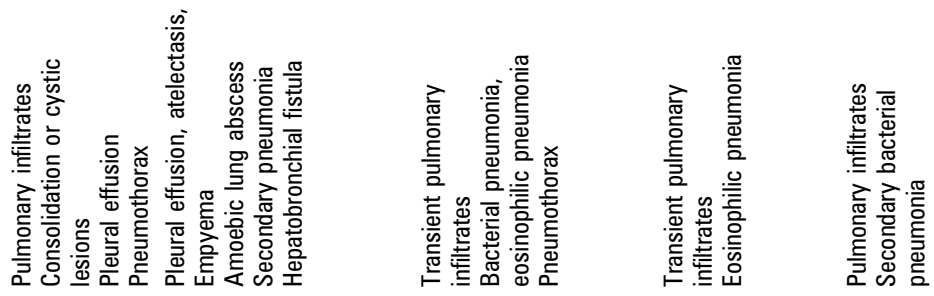

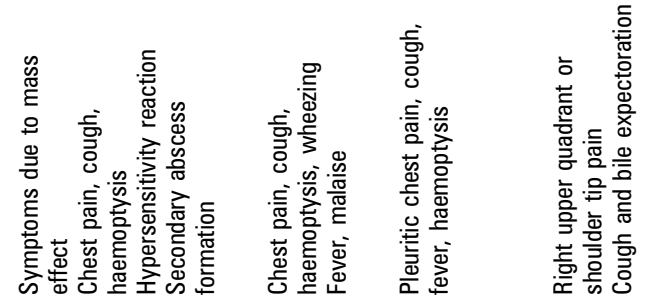
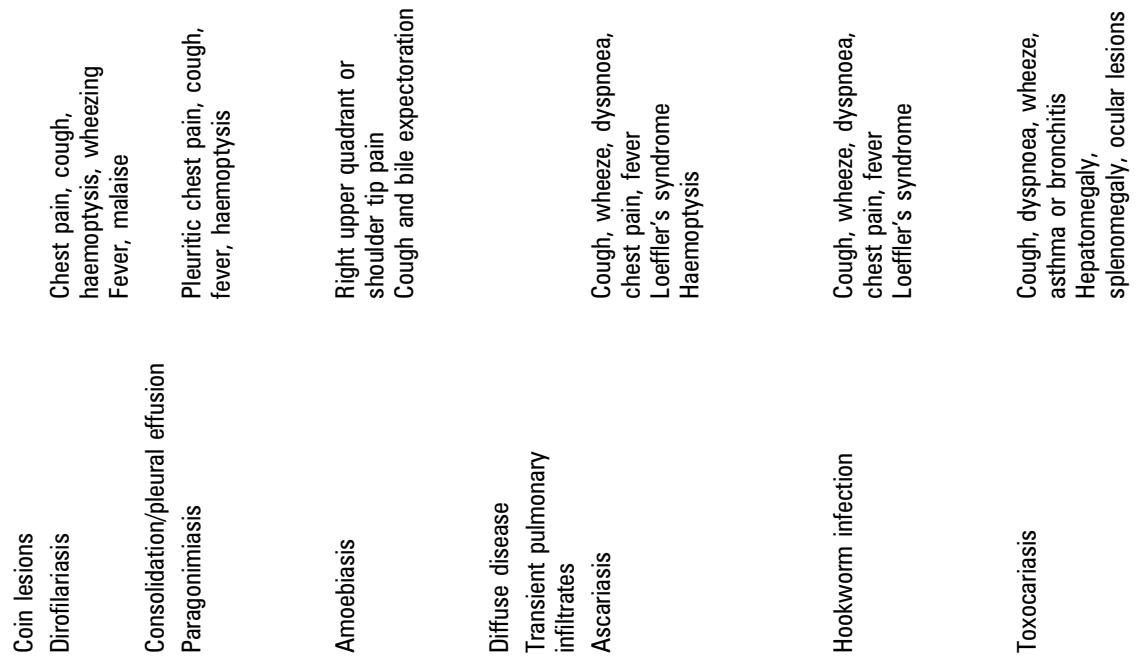

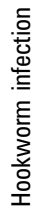

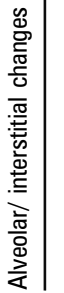




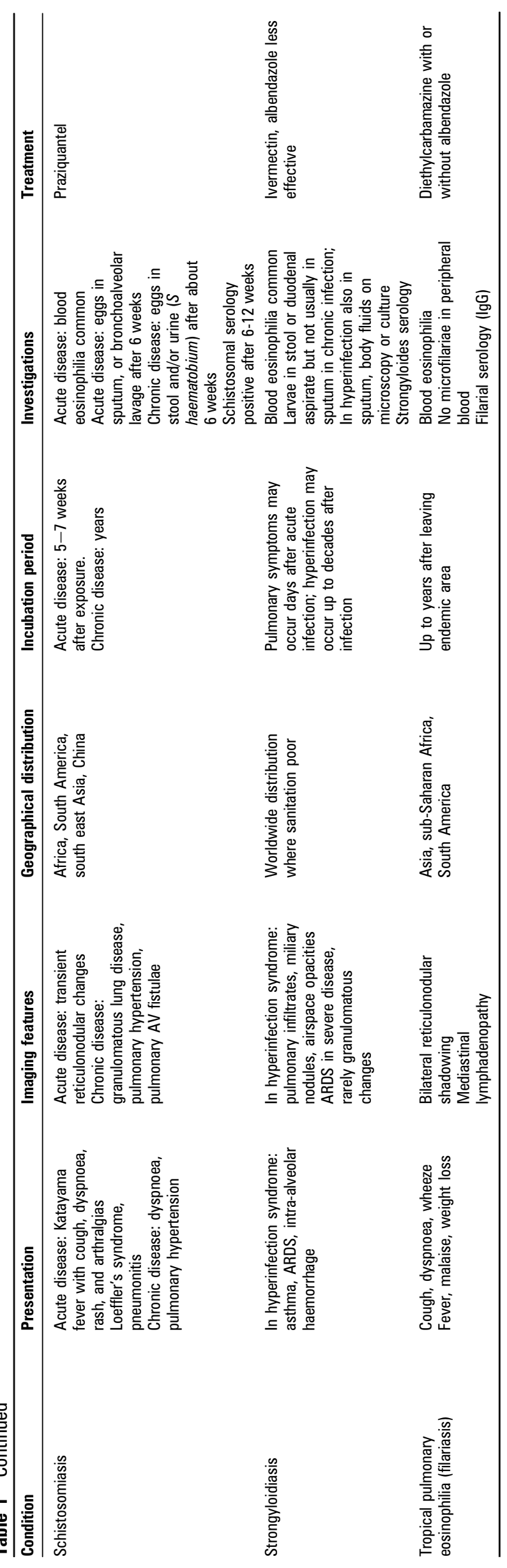

therapy), a hyperinfection syndrome can result in severe disseminated infection which may cause Gram-negative sepsis and death. Respiratory symptoms may be non-specific and, apart from gastrointestinal complaints, symptoms of asthma might be the only feature. In patients with new onset bronchial asthma or worsening of asthmatic episodes concurrent with immunosuppression, $S$ stercoralis infection should be considered, especially in patients from endemic countries. ${ }^{49}{ }^{50}$ In immunosuppressed patients with hyperinfection, symptoms and signs consistent with adult respiratory distress syndrome may be seen. ${ }^{51} 52$ In disseminated disease, extensive intra-alveolar haemorrhage has been reported and should be considered as a cause of acute deterioration. ${ }^{53}$

\section{Radiological findings}

In Strongyloides hyperinfection, pulmonary infiltrates are the most frequent feature seen on chest radiography. Radiographic changes may include miliary nodules, reticular opacities and airspace opacities ranging from multifocal to lobar distribution. These are thought to be due to secondary infection, haemorrhage, inflammatory pneumonitis and bacterial abscess formation. ${ }^{13}{ }^{52}$ If widespread air space shadowing is seen on chest radiography, adult respiratory distress syndrome should be strongly suspected. Rare manifestations of pulmonary strongyloidiasis include granulomatous changes leading to pulmonary fibrosis. ${ }^{54}$

\section{Laboratory investigations}

Blood eosinophilia is seen in more than $75 \%$ of patients with chronic infection but may be absent in the hyperinfection syndrome in immunocompromised patients. ${ }^{55} \mathrm{~A}$ definitive diagnosis is made by demonstration of rhabditiform larvae in stool, sputum or duodenal aspirates. Filariform larvae may also be seen. Larvae may be identified on direct microscopy but specialised culture techniques are more likely to yield positive results. Antibody assays have sensitivities of approximately $90 \%$; however, cross-reactions occur with other helminth infections and antibody levels may be low in immunocompromised patients.

\section{Management}

Treatment with ivermectin is usually effective. In cases of hyperinfection, repeated treatments are required and expert parasitological advice should be sought. ${ }^{28} 56-58$

\section{Tropical pulmonary eosinophilia Presentation}

Tropical pulmonary eosinophilia (TPE) typically results from a hypersensitivity reaction to the lymphatic filarial parasites Wuchereria bancrofti and Brugia malayi found in endemic regions of south east Asia, India, China and Africa. The most common clinical manifestation of filariasis is elephantiasis resulting from filarial obstruction of lymphatic vessels. The syndrome of TPE is thought to be an immunological response to microfilariae rather than acute infection. Clinically, TPE typically has slow onset over several months, with respiratory symptoms including cough, dyspnoea and wheeze which may be worse at night. Systemic features including fever, malaise and weight loss may occur. By contrast, Loeffler's syndrome is caused by larval migration of nematodes such as Strongyloides, Ascaris or hookworm which results in wheeze, cough and dyspnoea of acute onset and rarely lasts more than a few weeks.

\section{Radiological findings}

Bilateral fine nodular or reticulonodular shadowing have been described on plain chest radiography. ${ }^{59}$ However, it has been suggested that a CT scan of the thorax might be more sensitive 
to detect reticulonodular pulmonary opacities and mediastinal lymphadenopathy. ${ }^{60}$

\section{Laboratory investigations}

High titres of antifilarial antibodies and peripheral blood eosinophilia are present. The extensive immune response leads to rapid clearance of microfilariae from the blood and, in order to diagnose TPE, it is essential that no microfilariae can be found in the peripheral blood. Total serum IgE is raised, as are filarialspecific $\operatorname{IgE}$ and $\operatorname{IgG}$ antibody levels. Bronchoalveolar lavage may show evidence of an eosinophilic alveolitis with a high concentration of eosinophils. ${ }^{61}$ Specific antifilarial IgE and IgG antibodies have also been detected in bronchoalveolar lavage samples. ${ }^{62}$

\section{Management}

Pulmonary abnormalities usually progressively worsen without treatment. Concomitant infection with other filarial species must be investigated prior to treatment. Treatment is usually with diethylcarbamazine under expert guidance; however, it is sometimes given in combination with albendazole to improve efficacy. ${ }^{63} 64$ Even after treatment, pulmonary symptoms may progress and interstitial lung disease might develop leading to chronic respiratory failure. ${ }^{65}$

\section{Non-infectious causes of pulmonary eosinophilia}

Other causes of pulmonary eosinophilia need to be considered in the differential diagnosis of parasitic lung infections. Idiopathic lung diseases such as acute eosinophilic pneumonia, chronic eosinophilic pneumonia and idiopathic hypereosinophilic syndrome and lung diseases of known cause such as allergic bronchopulmonary aspergillosis, bronchocentric granulomatosis, drug reactions and eosinophilic vasculitis (Churg-Strauss syndrome) can cause pulmonary and peripheral blood eosinophilia. ${ }^{66} 67$

\section{CONCLUSIONS}

This review outlines the symptoms, signs, investigation and treatment of parasitic lung diseases which are summarised in table 1. The clinical presentation and radiology of many of these diseases may share common features with tuberculosis and malignancy. When symptoms of lung disease are associated with gastrointestinal, hepatobiliary or cutaneous symptoms or a peripheral eosinophilia, there should be a high index of suspicion for parasitic diseases. With increasing travel and migration, it is important to consider parasitic infections in the differential diagnosis of lung diseases. Parasitic lung infections have been described in patients following chemotherapy or transplantation and need to be considered in the differential diagnosis of lung infections in immunocompromised individuals. ${ }^{68-70}$ Direct identification of the causative organisms may be achieved definitively through microscopic examination of stool or respiratory tract samples, or indirectly via serological testing. If identified early, most parasitic lung diseases are curable with medical treatment. Further information on the distribution of parasitic lung diseases and treatment may be obtained from the World Health Organization. ${ }^{28} 71$

Funding PLC and AG are supported by the UCL Hospitals Comprehensive Biomedical Research Centre Infection Theme. AG was supported by a Public Health Career Scientist award from the UK Department of Health.

Competing interests None.

Provenance and peer review Not commissioned; externally peer reviewed.

\section{REFERENCES}

1. Gottstein B, Reichen J. Hydatid lung disease (echinococcosis/hydatidosis). Clin Chest Med 2002:23:397-408, ix.
2. Beggs I. The radiology of hydatid disease. AJR Am J Roentgenol 1985;145:639-48.

3. Erdem CZ, Erdem LO. Radiological characteristics of pulmonary hydatid disease in children: less common radiological appearances. Eur J Radiol 2003;45:123-8.

4. Tor M, Ozvaran K, Ersoy Y, et al. Pitfalls in the diagnosis of complicated pulmonary hydatid disease. Respir Med 2001;95:237-9.

5. Doiz 0, Benito R, Sbihi Y, et al. Western blot applied to the diagnosis and posttreatment monitoring of human hydatidosis. Diagn Microbiol Infect Dis 2001:41:139-42

6. Keshmiri M, Baharvahdat $\mathrm{H}$, Fattahi SH, et al. A placebo controlled study of albendazole in the treatment of pulmonary echinococcosis. Eur Respir $J$ 1999;14:503-7.

7. Shalabi RI, Ayed AK, Amin M. 15 Years in surgical management of pulmonary hydatidosis. Ann Thorac Cardiovasc Surg 2002;8:131-4.

8. Stamatakos M, Sargedi C, Stefanaki C, et al. Anthelminthic treatment: an adjuvant therapeutic strategy against Echinococcus granulosus. Parasitol Int 2009;58:115-20.

9. Brunetti E, Kern P, Vuitton DA. Expert consensus for the diagnosis and treatment of cystic and alveolar echinococcosis in humans. Acta Trop 2010;114:1-16.

10. Mawhorter S, Temeck B, Chang R, et al. Nonsurgical therapy for pulmonary hydatid cyst disease. Chest 1997;112:1432-6.

11. World Health Organization. Cestodes (tapeworm) - echinococcosis. WHO Essential Medicines Library, EMLib. 2009. http://search.who.int/search?q=PAIR\% 20AND\%20lung\%20 cysts\&spell $=1$ gaccess $=$ p\&output $=x \mathrm{ml}$ no dtd\&ie $=$ utf8\&client $=$ WHO\&sitesearch $=$ \&site $=$ default collection\&oe $=$ UTF-8qproxystylesheet $=$ WHO.

12. Bielawski BC, Harrington D, Joseph E. A solitary pulmonary nodule with zoonotic implications. Chest 2001;119:1250-2.

13. Armstrong P, Wilson A, Dee P, et al. Imaging of diseases of the chest. 3rd edn. St Louis: Mosby, 2000.

14. Moore W, Franceschi D. PET findings in pulmonary dirofilariasis. J Thorac Imaging 2005;20:305-6.

15. Chitkara RK, Sarinas PS. Dirofilaria, visceral larva migrans, and tropical pulmonary eosinophilia. Semin Respir Infect 1997:12:138-48.

16. Velez ID, Ortega JE, Velasquez LE. Paragonimiasis: a view from Columbia. Clin Chest Med 2002;23:421- $\mathrm{x}$.

17. Im JG, Whang HY, Kim WS, et al. Pleuropulmonary paragonimiasis: radiologic findings in 71 patients. AJR Am J Roentgenol 1992;159:39-43.

18. Mukae H, Taniguchi H, Matsumoto N, et al. Clinicoradiologic features of pleuropulmonary Paragonimus westermani on Kyusyu Island, Japan. Chest 2001;:120:514-20.

19. Jeon $\mathbf{K}$, Koh WJ, Kim H, et al. Clinical features of recently diagnosed pulmonary paragonimiasis in Korea. Chest 2005;128:1423-30.

20. Nakamura-Uchiyama F, Mukae H, Nawa Y. Paragonimiasis: a Japanese perspective. Clin Chest Med 2002;23:409-20.

21. Watanabe S, Nakamura $Y$, Kariatsumari $K$, et al. Pulmonary paragonimiasis mimicking lung cancer on FDG-PET imaging. Anticancer Res 2003;23:3437-40.

22. Tomita M, Matsuzaki $Y$, Nawa $Y$, et al. Pulmonary paragonimiasis referred to the department of surgery. Ann Thorac Cardiovasc Surg 2000;6:295-8.

23. Mukae 0, Taniguchi H, Ashitani J, et al. Case report: Paragonimiasis westermani with seroconversion from immunoglobulin (Ig) $\mathrm{M}$ to $\lg \mathrm{G}$ antibody with the clinical course. Am J Trop Med Hyg 2001;65:837-9.

24. DeFrain M, Hooker R. North American paragonimiasis: case report of a severe clinical infection. Chest 2002;121:1368-72.

25. Mandell, Douglas and Bennett's Principles and Practice of Infectious Diseases. 7th edn. Churchill Livingstone, Elsevier, 2009.

26. Cohen J, Powderly WG. Infectious diseases. London: Elsevier Mosby, 2010

27. Cook G. Manson's tropical diseases. Philadelphia: WB Saunders, 2002.

28. WHO Essential Medicines Library. 2009. http://www.who.int/medicines/publications/ essentialmedicines/en/.

29. Badalamenti S, Jameson JE, Reddy KR. Amebiasis. Curr Treat Options Gastroenterol 1999;2:97-103.

30. Sarinas PS, Chitkara RK. Ascariasis and hookworm. Semin Respir Infect 1997:12:130-7.

31. Santini M, Baldi A, Vicidomini G, et al. Relapsing pneumothorax due to Ascaris lumbricoides larvae. Monaldi Arch Chest Dis 1999;54:328-9.

32. Inoue $\mathbf{K}$, Inoue $\mathrm{Y}$, Arai $\mathrm{T}$, et al. Chronic eosinophilic pneumonia due to visceral larva migrans. Intern Med 2002;41:478-82.

33. Roig J, Romeu J, Riera C, et al. Acute eosinophilic pneumonia due to toxocariasis with bronchoalveolar lavage findings. Chest 1992:102:294-6.

34. Dubinsky P, Akao N, Reiterova K, et al. Comparison of the sensitive screening kit with two ELISA sets for detection of anti-Toxocara antibodies. Southeast Asian J Trop Med Public Health 2000;31:394-8.

35. van der Werf MJ, de Vlas SJ, Brooker S, et al. Quantification of clinical morbidity associated with schistosome infection in sub-Saharan Africa. Acta Trop 2003;86:125-39

36. Ross AG, Vickers D, Olds GR, et al. Katayama syndrome. Lancet Infect Dis 2007:7:218-24.

37. Barbosa MM, Lamounier JA, Oliveira EC, et al. Pulmonary hypertension in schistosomiasis mansoni. Trans $R$ Soc Trop Med Hyg 1996;90:663-5.

38. Bethlem EP, Schettino GP, Carvalho CR. Pulmonary schistosomiasis. Curr Opin Pulm Med 1997; 3:361-5

39. Schwartz E, Rozenman J, Perelman M. Pulmonary manifestations of early schistosome infection among nonimmune travelers. Am J Med 2000;109:718-22. 
40. Schaberg T, Rahn W, Racz P, et al. Pulmonary schistosomiasis resembling acute pulmonary tuberculosis. Eur Respir J 1991;4:1023-6.

41. Lambertucci JR, Silva LC, de Queiroz LC. Pulmonary nodules and pleural effusion in the acute phase of Schistosomiasis mansoni. Rev Soc Bras Med Trop 2007:40:374-5

42. Waldman AD, Day JH, Shaw $\mathrm{P}$, et al. Subacute pulmonary granulomatous schistosomiasis: high resolution CT appearances-another cause of the halo sign. $\mathrm{Br}$ $J$ Radiol 2001;74:1052-5.

43. Day JH, Grant $A D$, Doherty JF, et al. Schistosomiasis in travellers returning from sub-Saharan Africa. BMJ 1996;313:268-9.

44. Doehring E, Feldmeier H, Daffalla AA. Day-to-day variation and circadian rhythm of egg excretion in urinary schistosomiasis in the Sudan. Ann Trop Med Parasitol 1983;77:587-94.

45. Sarda RK, Minjas JN, Mahikwano LF. Further observations on the use of gross haematuria as an indirect screening technique for the detection of Schistosoma haematobium infection in school children in Dar es Salaam, Tanzania. J Trop Med Hyg 1986:89:309-12.

46. Tosswill JH, Ridley DS. An evaluation of the ELISA for schistosomiasis in a hospital population. Trans R Soc Trop Med Hyg 1986;80:435-8.

47. Doherty JF, Moody AH, Wright SG. Katayama fever: an acute manifestation of schistosomiasis. BMJ 1996; 313:1071-2.

48. Hall A, Nokes C, Wen ST, et al. Alternatives to bodyweight for estimating the dose of praziquantel needed to treat schistosomiasis. Trans $R$ Soc Trop Med Hyg 1999;93:653-8.

49. Higenbottam TW, Heard BE. Opportunistic pulmonary strongyloidiasis complicating asthma treated with steroids. Thorax 1976;31:226-33.

50. Sen P, Gil C, Estrellas B, et al. Corticosteroid-induced asthma: a manifestation of limited hyperinfection syndrome due to Strongyloides stercoralis. South Med J 1995;88:923-7.

51. Thompson JR, Berger R. Fatal adult respiratory distress syndrome following successful treatment of pulmonary strongyloidiasis. Chest 1991;99:772-4.

52. Woodring $\mathbf{J H}$, Halffill H, Reed JC. Pulmonary strongyloidiasis: clinical and imaging features. AJR Am J Roentgenol 1994;162:537-42.

53. Kinjo T, Tsuhako K, Nakazato I, et al. Extensive intra-alveolar haemorrhage caused by disseminated strongyloidiasis. Int $J$ Parasitol 1998;28:323-30.

54. Lin AL, Kessimian N, Benditt JO. Restrictive pulmonary disease due to interlobular septal fibrosis associated with disseminated infection by Strongyloides stercoralis. Am J Respir Crit Care Med 1995;151:205-9.

55. Woodring $\mathbf{J H}$, Halfhill $\mathrm{H}$, Berger $\mathrm{R}$, et al. Clinical and imaging features of pulmonary strongyloidiasis. South Med J 1996;89:10-19.
56. Chiodini PL, Reid AJ, Wiselka MJ, et al. Parenteral ivermectin in Strongyloides hyperinfection. Lancet 2000;355:43-4.

57. Marti H, Haji HJ, Savioli L, et al. A comparative trial of a single-dose ivermectin versus three days of albendazole for treatment of Strongyloides stercoralis and other soil-transmitted helminth infections in children. Am J Trop Med Hyg 1996:55:477-81.

58. Zaha 0, Hirata T, Kinjo F, et al. Strongyloidiasis: progress in diagnosis and treatment. Intern Med 2000;39:695-700

59. Udwadia FE. Tropical eosinophilia: a review. Respir Med 1993:87:17-21.

60. Sandhu M, Mukhopadhyay S, Sharma SK. Tropical pulmonary eosinophilia: a comparative evaluation of plain chest radiography and computed tomography. Australas Radiol 1996:40:32-7.

61. Vijayan VK, Sankaran K, Venkatesan P, et al. Effect of diethylcarbamazine on the alveolitis of tropical eosinophilia. Respiration 1991;58:255-9.

62. Nutman TB, Vijayan VK, Pinkston P, et al. Tropical pulmonary eosinophilia: analysis of antifilarial antibody localized to the lung. J Infect Dis 1989;160:1042-50.

63. McLaughlin SI, Radday J, Michel MC, et al. Frequency, severity, and costs of adverse reactions following mass treatment for lymphatic filariasis using diethylcarbamazine and albendazole in Leogane, Haiti, 2000. Am J Trop Med Hyg 2003:68:568-73

64. Ramzy RM, el Setouhy M, Helmy H, et al. The impact of single-dose diethylcarbamazine treatment of bancroftian filariasis in a low-endemicity setting in Egypt. Am J Trop Med Hyg 2002:67:196-200.

65. Rom WN, Vijayan VK, Cornelius MJ, et al. Persistent lower respiratory tract inflammation associated with interstitial lung disease in patients with tropical pulmonary eosinophilia following conventional treatment with diethylcarbamazine. Am Rev Respir Dis 1990;142:1088-92.

66. Jeong YJ, Kim Kl, Seo IJ, et al. Eosinophilic lung diseases: a clinical, radiologic, and pathologic overview. Radiographics 2007;27:617-37.

67. Janz DR, O'Neal HR Jr, Ely EW. Acute eosinophilic pneumonia: a case report and review of the literature. Crit Care Med 2009:37:1470-4.

68. Balagopal A, Mills L, Shah A, et al. Detection and treatment of Strongyloides hyperinfection syndrome following lung transplantation. Transpl Infect Dis 2009;11:149-54.

69. Gupta S, Jain A, Fanning TV, et al. An unusual cause of alveolar hemorrhage pos hematopoietic stem cell transplantation: a case report. BMC Cancer 2006;6:87.

70. de Gorgolas M, Casado V, Renedo G, et al. Nodular lung schistosomiais lesions after chemotherapy for dysgerminoma. Am J Trop Med Hyg 2009;81:424-7.

71. World Health Organization. Parasitic diseases and epidemiology. 2009. http://www.who.int/topics/tropical_diseases/en/. 